\title{
Poster session II
}

CORONAL MASS EJECTIONS

INTERPLANETARY CORONAL MASS EJECTIONS

SPACE WEATHER 


\title{
Topological study of active region 11158
}

\author{
Jie Zhao ${ }^{1,2}$, Hui Li ${ }^{1,2}$, Etienne Pariat ${ }^{3}$, Brigitte Schmieder ${ }^{3}$, \\ Yang Guo ${ }^{4}$ and Thomas Wiegelmann ${ }^{5}$ \\ ${ }^{1}$ Purple Mountain Observatory, CAS, 2 West Beijing Road, Nanjing 210008, China \\ ${ }^{2}$ Key Laboratory of Dark Matter and Space Astronomy, CAS, Nanjing 210008, China \\ ${ }^{3}$ LESIA, Observatoire de Paris, Section de Meudon, F-92195,Meudon Principal Cedex, France \\ ${ }^{4}$ School of Astronomy \& Space Science, Nanjing University, Nanjing 210093, China \\ ${ }^{5}$ Max-Planck-Institut für Sonnensystemforschung, Max-Planck-Strasse 2, 37191 \\ Katlenburg-Lindau, Germany \\ email: nj.lihui@pmo.ac.cn
}

\begin{abstract}
With the cylindrical equal area (CEA) projection data from the Helioseismic and Magnetic Imager (HMI) onboard the Solar Dynamics Observatory (SDO), we reconstructed the three-dimensional (3D) magnetic fields in the corona ,using a non-linear force-free field (NLFFF) extrapolation method every 12 minutes during five days, to calculate the squashing degree factor $Q$ in the volume. The results show that this AR has an hyperbolic flux tube (HFT) configuration, a typical topology of quadrupole, which is stable even during the two large flares (M6.6 and X2.2 class flares).
\end{abstract}

Keywords. corona, flares, magnetic topology

\section{Introduction}

The different behaviors of solar atmosphere in response to the plasma motions and instabilities are thought to be mainly due to the different topologies in the coronal magnetic field (Berger 1991). Like null point exists at cross section of separatrices, there is hyperbolic flux tube (HFT) at cross section of quasi-separatrix layers (QSLs). Strong electric currents, which dynamically form in non-potential magnetic field, are physically responsible for flaring (Aulanier et al. 2005). QSLs, especially the HFTs, are crucial for a region to flare as they are preferential places for narrow current sheet formation (Titov et al. 2002). We focus on the topological analysis of active region (AR) 11158 in this work. The method used to calculate $\mathrm{Q}$ values is introduced in Section 2 and we discuss our results in Section 3.

\section{Computational method of QSLs}

We use the equations proposed in Pariat et al. (2012)

$$
\begin{aligned}
& \left(\left(d_{X 2 x_{c}} d_{y 1 y_{c}}-d_{X 2 y_{c}} d_{y 1 x_{c}}\right)^{2}\right. \\
Q_{m 3}= & +\left(d_{X 2 y_{c}} d_{x 1 x_{c}}-d_{X 2 x_{c}} d_{x 1 y_{c}}\right)^{2} \\
& +\left(d_{Y 2 x_{c}} d_{y 1 y_{c}}-d_{Y 2 y_{c}} d_{y 1 x_{c}}\right)^{2} \\
& \left.+\left(d_{Y 2 y_{c}} d_{x 1 x_{c}}-d_{Y 2 x_{c}} d_{x 1 y_{c}}\right)^{2}\right) f
\end{aligned}
$$

with

$$
f=\frac{\left|B_{z, 1}\left(x_{1}, y_{1}\right) B_{Z, 2}\left(X_{2}, Y_{2}\right)\right|}{\left|B_{n, c}\left(x_{c}, y_{c}\right)\right|^{2} \delta^{4}}
$$

to calculate the $\mathrm{Q}$ value at $\left(x_{c}, y_{c}\right)$ on a fix plane. 


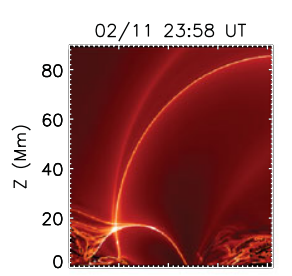

02/13 11:58 UT

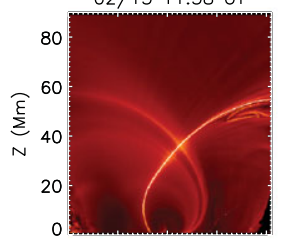

02/14 23:58 UT

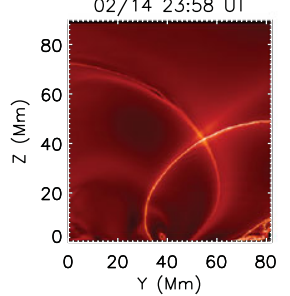

$02 / 12 \quad 11: 58$ UT

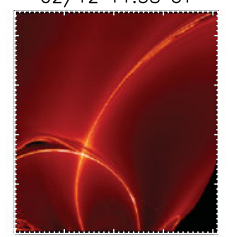

$02 / 13 \quad 23: 58$ UT

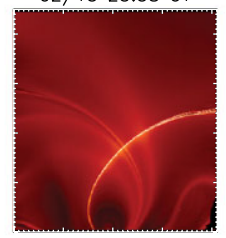

$02 / 1511: 58$ UT

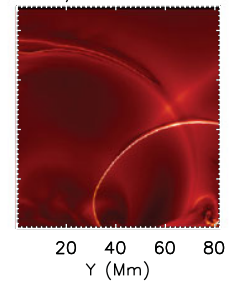

$02 / 12$ 23:58 UT

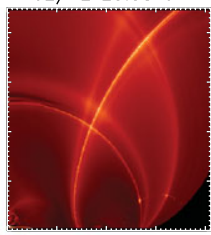

$02 / 14 \quad 11: 58$ UT

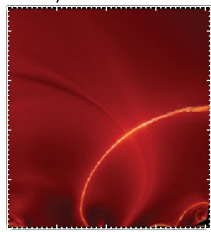

$02 / 15$ 23:58 UT

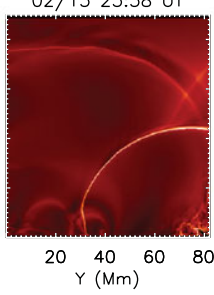

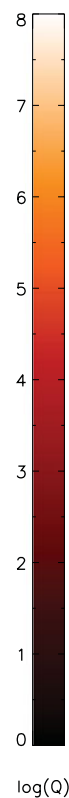

Figure 1. Evolution of $\mathrm{Q}$ maps in a vertical cut. The $\mathrm{Q}$ value is displayed in logarithm scale and time cadence between two successive panels is 12 hours.

The reference boundary here is chosen to be the photosphere. The parameters $d_{x 1 x_{c}}$, $d_{y 1 x_{c}}, d_{x 1 y_{c}}, d_{y 1 y_{c}}, d_{X 2 x_{c}}, d_{Y 2 x_{c}}, d_{X 2 y_{c}}, d_{Y 2 y_{c}}$ are the components of the field lines footpoint distance on the photosphere. These field lines pass through the four surrounding points of $\left(x_{c}, y_{c}\right)$ on the fix plane. $B_{z, 1}\left(x_{1}, y_{1}\right)$ and $B_{Z, 2}\left(X_{2}, Y_{2}\right)$ are the vertical components of magnetic field at the two footpoints $\left(x_{1}, y_{1}\right)$ and $\left(X_{2}, Y_{2}\right), B_{n, c}\left(x_{c}, y_{c}\right)$ is the value of the magnetic field component normal to the fix plane at $\left(x_{c}, y_{c}\right)$.

\section{Results and discussion}

From the temporal series of Q maps in 3D, we noticed that the quadrupole is relatively stable, which was there even during the large flares. Large-scale HFT always exists and is stable for 36 hours since 11:58 UT of 13 February. Titov et al. (2003) has studied this kind of large-scale HFT structure and suggested that the magnetic pinching inside caused by the large-scale shearing motion on the photosphere could produce a large flare. However, in our case, we do not find large flare directly related to this HFT. We suggest that it will be hard to be distinguished from the visible flare ribbons although the possible reconnection at this place may induce the ultra-violet (UV) enhancement at the intersection of HFT configuration and the photosphere.

\section{References}

Aulanier, G., Pariat, E., \& Démoulin, P. 2005, A\&A, 444, 961

Berger, M. A. 1991, Advances in Solar System Magnetohydrodynamics, 241

Pariat, E. \& Démoulin, P. 2012, A\&A, 541, A78

Titov, V. S., Hornig, G., \& Démoulin, P. 2002, JGR, 107, 1164

Titov, V. S., Galsgaard, K., \& Neukirch, T. 2003, ApJ, 582, 1172 